\title{
On the Entangling Power of Quantum Evolutions
}

\author{
Paolo Zanardi ${ }^{1,3}$, Christof Zalka ${ }^{1}$ and Lara Faoro ${ }^{2,3}$ \\ 1 Institute for Scientific Interchange (ISI) Foundation, Viale Settimio Severo 65, I-10133 Torino, Italy \\ ${ }^{2}$ Dipartimento di Fisica Politecnico di Torino, Viale Duca degli Abruzzi 24, I-10129 Torino, Italy \\ ${ }^{3}$ Istituto Nazionale per la Fisica della Materia (INFM)
}

(August 11, 2018)

\begin{abstract}
We analyze the entangling capabilities of unitary transformations $U$ acting on a bipartite $d_{1} \times d_{2^{-}}$ dimensional quantum system. To this aim we introduce an entangling power measure $e(U)$ given by the mean linear entropy produced acting with $U$ on a given distribution of pure product states. This measure admits a natural interpretation in terms of quantum operations. For a uniform distribution explicit analytical results are obtained using group-theoretic arguments. The behavior of the features of $e(U)$ as the subsystem dimensions $d_{1}$ and $d_{2}$ are varied is studied both analytically and numerically. The two-qubit case $d_{1}=d_{2}=2$ is argued to be peculiar.
\end{abstract}

PACS numbers: 03.67.Lx, 03.65.Fd

From the beginning it has been argued that entanglement is one of the crucial ingredients that allows Quantum Information processing [1] to outperform, for certain tasks, any classically operating device. In this sense entanglement represents a uniquely quantum resource whose production is a sort of elementary prerequisite for any Quantum Computation (QC). Such a basic task is accomplished by unitary transformations $U$ i.e., quantum evolutions acting on the state-space of the multi-partite system that describe non-trivial interactions between the degrees of freedom of the different subsystems. Even though almost all the unitaries satisfy this latter requirement [2], it is quite natural to ask how different $U$ 's are efficient, according to some criterion to be specified, as entanglers, and then by using such a criterion to analyze the full manifold of bi-partite quantum evolutions.

In this paper we address this issue by introducing over the space of bi-partite unitaries a measure for their entangling power. This is done by considering how much entanglement is produced by $U$ on the average acting on a given distribution of unentangled quantum states. The kind of situation we have in mind is a procedure for entanglement production in which one randomly generates product states (the "cheap" resource ) according to some probability distribution $p$ and then applies the transformation $U$. The average entanglement obtained with the above scheme will be our measure $e_{p}(U)$ of the quantum evolution $U$.

It is important to stress that these U's can represent different objects, both from the logical and physical point of view. Some prototypical instances are given by: a) A quantum computation using a pair of quantum registers. Here the entangling power measure will quantify how the computation $U$ is efficient in making the first (say memory) and the second (say computational) registers entangled. This kind of entanglement, that represents mutual information between the two registers, has been recently proved to play a role in $\mathrm{QC}$ viewed as a communication process [3]. b) The global evolution of a system plus its environment. In this case $e_{p}(U)$ measures the decohering power of the system-environment coupled evolution $U$. Engineering weak decoherence then amounts to design an optimal $U$ with respect to the criterion of minimal entangling power. c) A single two-subsystem e.g., twoqubits, gate in a quantum-network. Now the entangling $U$ are the two-qubit gates needed to get universal QC [2].

To formalize our setting let us consider a bipartite quantum system with state space $\mathcal{H}=\mathcal{H}_{1} \otimes \mathcal{H}_{2}$ where $\operatorname{dim} \mathcal{H}_{i}=d_{i}(i=1,2)$ and $U \in \mathcal{U}(\mathcal{H}) \cong U\left(d_{1} d_{2}\right)$. If $E$ is an entanglement measure over $\mathcal{H}$ we define the entangling power of $U$ (with respect to $E$ ) as

$$
e_{p}(U):=\overline{E\left(U\left|\psi_{1}\right\rangle \otimes\left|\psi_{2}\right\rangle\right)} \psi_{1}, \psi_{2}
$$

where the bar denotes the average over to all the product states $\left|\psi_{1}\right\rangle \otimes\left|\psi_{2}\right\rangle$. distributed according some probability density $p\left(\psi_{1}, \psi_{2}\right)$ over the manifold of product states.

We shall use as entanglement measure of $|\Psi\rangle \in \mathcal{H}$ the linear entropy

$$
E(|\Psi\rangle):=1-\operatorname{tr}_{1} \rho^{2}, \rho:=\operatorname{tr}_{2}|\Psi\rangle\langle\Psi| .
$$

This quantity measures the purity of the reduced density matrix $\rho$, it can be regarded as a kind of "linearized" version of the von Neumann entropy $S(\rho)=-\operatorname{tr} \rho \ln \rho$, which is known to provide the essentially unique measure of entanglement for bi-partite pure quantum states. One has that $0 \leq E(|\Psi\rangle) \leq 1-1 / d$ where the lower (upper) bound is reached iff $|\psi\rangle$ is a product state (maximally entangled). The measure (2) has, with respect to $S(\rho)$, the definite advantage of being a polynomial in $|\psi\rangle$.

Now we introduce some notations. We shall denote by $T_{i j},(i, j=1, \ldots, 4)$ the transposition between the $i$-the and the $j$-th factor of $\mathcal{H}^{\otimes 2}:=\mathcal{H}_{2} \cong\left(\mathbb{C}^{d_{1}} \otimes \mathbb{C}^{d_{2}}\right) \otimes\left(\mathbb{C}^{d_{1}} \otimes\right.$ $\left.\mathbb{C}^{d_{2}}\right)$. Notice that $T_{12}$ and $T_{34}$ are well defined elements of $\mathcal{U}\left(\mathcal{H}^{\otimes 2}\right)$ only when $d_{1}=d_{2}$, in this latter case such operators will be referred to as swaps. Moreover - when $\mathcal{H}_{i} \cong \mathcal{H}_{j}$ - one defines the projectors $P_{i j}^{ \pm}:=2^{-1}\left(\mathbb{1} \pm T_{i j}\right)$ 
over the totally symmetric (antisymmetric) subspaces of $\mathcal{H}_{i} \otimes \mathcal{H}_{j}$, the latter being thought of as embedded in $\mathcal{H}^{\otimes 2}$. The space End $\left(\mathcal{H}^{\otimes 2}\right)$ is endowed with the HilbertSchmidt scalar product $\langle A, B\rangle:=\operatorname{tr}\left(A^{\dagger} B\right)$. Finally with $\mathcal{S}(\mathcal{H})$ we shall denote the space of density matrices over $\mathcal{H}$.

Proposition 0 The entangling power (11) is given by

$$
e_{p}(U)=2 \operatorname{tr}\left[U^{\otimes 2} \Omega_{p} U^{\dagger \otimes 2} P_{13}^{-}\right]
$$

where $\Omega_{p}:=\int d \mu\left(\psi_{1}, \psi_{2}\right)\left(\left|\psi_{1}\right\rangle\left\langle\psi_{1}|\otimes| \psi_{2}\right\rangle\left\langle\psi_{2}\right|\right)^{\otimes 2} \in$ $\mathcal{S}\left(\mathcal{H}^{\otimes 2}\right)$ and $d \mu$ denotes the measure over the product state manifold induced by the probability distribution $p\left(\psi_{1}, \psi_{2}\right)$.

Proof. Let us observe that Eq. (2) can be written in a linear form using the identity $\operatorname{tr}[(A \otimes B) T]=$ $\operatorname{tr}(A B)$ where $T$ is the swap. Then $E(|\Psi\rangle)=1-$ $\operatorname{tr}\left(|\Psi\rangle\left\langle\left.\Psi\right|^{\otimes 2} T_{13}\right)\right.$. Form this remark and the definition (1) it follows immediately (3)

The result above express $e_{p}(U)$ as the expectation value over $\Omega_{p}$ of the positive operator $2 U^{\dagger \otimes 2} P_{13}^{-} U^{\otimes 2}$. This latter operator can be viewed as the effect associated to the completely positive (CP) [4] map $\Phi_{U}$ on $\mathcal{S}\left(\mathcal{H}^{\otimes 2}\right)$ given by $\Phi_{U}: \Omega \mapsto 2 P_{13}^{-} U^{\otimes 2} \Omega U^{\dagger \otimes 2} P_{13}^{-}$. This remark allows us to interpret the entangling power (4) as probability of success of a two-party (A and B) quantum protocol (see Fig. 1). Suppose $A(B)$ owns spaces $\mathcal{H}_{1}$ and $\mathcal{H}_{3}\left(\mathcal{H}_{2}\right.$ and $\left.\mathcal{H}_{4}\right)$

a) A and B generate pairs of states $\left|\psi_{1}\right\rangle \otimes\left|\psi_{2}\right\rangle$ according the distribution probability $p\left(\psi_{1}, \psi_{2}\right)\left[\Omega_{p}\right.$ is prepared] b) Apply to each member of the pair the joint transformation $U$ [action of $U^{\otimes 2}$ ] c) Perform a projective measurement of $\sqrt{2} P_{13}^{-}$.

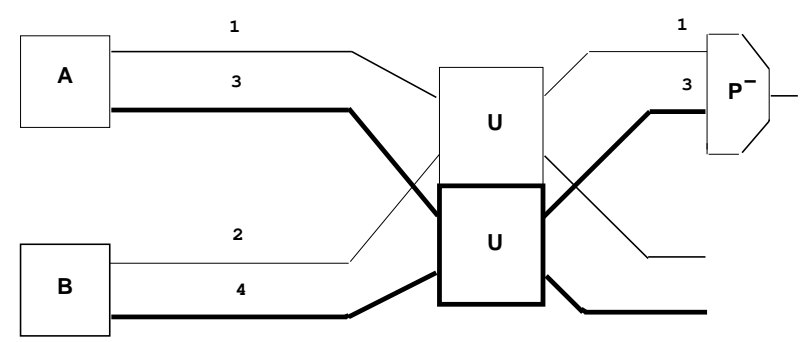

FIG. 1. Scheme of the two-party protocol realizing the operation $\Phi_{U}$.

Eq. (3) nicely displays several properties required for any entangling measure for bi-partite unitary evolutions. i) $e_{p}\left(U_{1} \otimes U_{2} U\right)=e_{p}(U)\left(U_{i} \in U\left(d_{i}\right)\right)$. Indeed from the $U\left(d_{1}\right)$-invariance of $P_{13}^{-}$one finds $e_{p}\left(U_{1} \otimes U_{2} U\right)=2 \operatorname{tr}\left[U^{\otimes 2} \Omega_{p} U^{\dagger \otimes 2}\left(U_{1} \otimes U_{2}\right)^{\dagger \otimes 2} P_{13}^{-}\left(U_{1} \otimes\right.\right.$ $\left.\left.U_{2}\right)^{\otimes 2}\right]=2 \operatorname{tr}\left[U^{\otimes 2} \Omega_{p} U^{\dagger \otimes 2}\left(U_{1}\right)_{13}^{\dagger} P_{13}^{-}\left(U_{1}\right)_{13}\right]=e_{p}(U)$.
Where $\left(U_{1}\right)_{13}:=U_{1} \otimes \mathbb{1} \otimes U_{1} \otimes \mathbb{1}$. ii) When $d_{1}=d_{2}$, by denoting with $T$ the transposition between the two factors of $\mathcal{H}$, one has $e_{p}(T U)=e_{p}(U)$. This stems from $T^{\otimes 2} P_{13}^{-} T^{\otimes 2}=P_{24}^{-}$. This leaves $e_{p}(U)$ unchanged, indeed this label change amounts simply to the replacement $\operatorname{tr}_{1} \leftrightarrow \operatorname{tr}_{2}$ in Eq. (2). Since, for pure states, the two reduced density matrices are isospectral the linear entropy is unchanged. Moreover if $\Omega_{p}$ is swap invariant i.e., $p\left(\psi_{1}, \psi_{2}\right)=p\left(\psi_{2}, \psi_{1}\right)$ one also has $e_{p}(U T)=e_{p}(U)$. iii) One has $e_{p}(\mathbb{1})=0$. This simply because $\Omega_{p} P_{13}^{-}=0$. Indeed, form the definition (3) one has $\Omega_{p} T_{13}=\Omega_{p}$. iv) From the previous remarks it follows that the entangling power is constant along the orbits in $\mathcal{U}(\mathcal{H})$ of the left action of the subgroup of the bi-local operations $U_{1} \otimes U_{2}$. In particular $e$ vanishes on all the elements of such a group. In the symmetric case $d_{1}=d_{2}$ the group is extended by the swap $T$.

Different distributions $p\left(\psi_{1}, \psi_{2}\right)$ would result in very different $e(U)$. An extreme example of this obvious remark is provided by transformations $U$ that simply permutes elements of a given basis $|i\rangle \otimes|j\rangle$ of $\mathcal{H}$. If $p$ is supported just on this basis the associated $e_{p}(U)$ vanishes identically, while we shall show later for a different probability distribution that U's can even be maximally entangling. Another example is given in the context of the case b) mentioned in the introduction. Suppose $U$ admits a decoherence-free subspace $\mathcal{C} \subset \mathcal{H}_{1}$ [5] the if $p$ is, for any $\left|\psi_{2}\right\rangle$, supported in $\mathcal{C}$ then again $e_{p}(U)=0$.

From now on we focus on the case in which $p$ is the uniform distribution $p_{0}$. With this term we refer to the unique $U\left(d_{1}\right) \times U\left(d_{2}\right)$-invariant probability distribution i.e., $p\left(\psi_{1}, \psi_{2}\right)=p\left(U_{1} \psi_{1}, U_{2} \psi_{2}\right)$. When all the product state are considered to be equally easy to be prepared, this latter assumption on $p$ is quite natural from the physical point of view [6]. Moreover, in view of its symmetry, the uniform $p$ will result in a great computational simplification that will allow for an explicit analytical evaluation of the average over the product-state manifold that appears in Eq. (11).

Let us begin by proving an easy group-theoretic Lemma that will play an essential technical role in the following.

Lemma $\Omega_{p_{0}}=4 C_{d_{1}} C_{d_{2}} P_{13}^{+} P_{24}^{+}, C_{d}^{-1}:=d(d+1)$.

Proof. Since the uniform distribution factorizes we can consider separately the average $\omega_{13}$ with respect to $\left|\psi_{1}\right\rangle$ ( on the first and the third factor of $\mathcal{H}^{\otimes 2}$ ) and the one $\omega_{24}$ with respect to $\left|\psi_{2}\right\rangle$ ( on the second and the fourth factor of $\mathcal{H}^{\otimes 2}$ ) then $\Omega_{p_{0}}=\omega_{13} \omega_{24}$. Let first observe that in view of definition (3) one has that $\Omega_{p_{0}}$ is supported in $P_{13}^{+} P_{24}^{+} \mathcal{H}^{\otimes 2}$ i.e., $\Omega_{p}$ is symmetric under the exchange of the first (second) and the third (fourth) factor. Moreover since the uniform distribution is $U\left(d_{1}\right) \times U\left(d_{2}\right)$ invariant one has $\left[U_{1}^{\otimes 2}, \omega_{13}\right]=0, \forall U_{1} \in U\left(d_{1}\right)$, and analogously for $\omega_{24}$. Since the $U_{1}^{\otimes 2}$, s act on the totally symmetric subspace irreducibly, it follows from the above commutation 
relation and the Schur Lemma [7] that $\omega_{13}=2 C P_{13}^{+}$. The normalization constant is found by the condition $\operatorname{tr} \omega_{13}=1$. Reasoning in the same way for $\omega_{24}$ one gets the desired result.

Proposition 1 The entangling power (1), with respect the uniform distribution, is given by

$$
\begin{aligned}
e_{p_{0}}(U) & =1-C_{d_{1}} C_{d_{2}} \sum_{\alpha=0,1} I_{\alpha}(U) \\
I_{\alpha}(U) & =t(\alpha)+<U^{\otimes 2}\left(T_{1+\alpha, 3+\alpha}\right) U^{\dagger \otimes 2}, T_{13}>,
\end{aligned}
$$

where $t(\alpha):=\operatorname{tr} T_{1+\alpha, 3+\alpha}$.

Proof. It is just a calculation. Insert $\Omega_{p_{0}}=$ $C_{d_{1}} C_{d_{2}}\left(\mathbb{1}+T_{13}\right)\left(\mathbb{1}+T_{24}\right)$ in Eq. (3). Notice that one has $\operatorname{tr} T_{13}=d_{1} d_{2}^{2}, \operatorname{tr} T_{24}=d_{1}^{2} d_{2}$.

From the relations $\left[T_{1+\alpha, 3+\alpha},\left(U_{1} \otimes U_{2}\right)^{\otimes 2}\right]=0,(\alpha=$ $0,1)$ it follows that both the functions $I_{0}$ and $I_{1}$ are invariant under the two-sided i.e.,left and right, action of bi-local unitaries e.g., $I_{1}(U)=I_{1}\left(U U_{1} \otimes U_{2}\right)$. Moreover in the symmetric case $d_{1}=d_{2}$ it follows from the above that the entangling power (4) can be written in a manifestly swap invariant form $e_{p_{0}}(U)=1-C_{d}^{2} \sum_{i=0}^{1} I_{d}\left(T^{i} U\right)$, where $I_{d}(U):=d^{3}+<U^{\otimes 2}, T_{13} U^{\otimes 2} T_{13}>$.

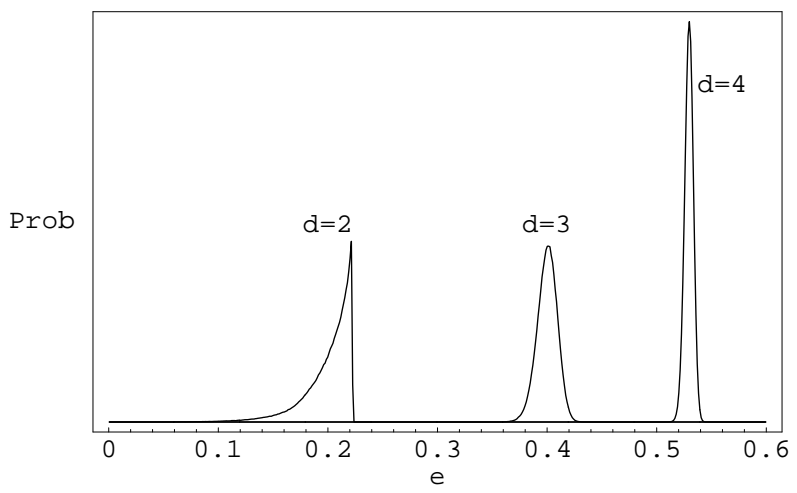

FIG. 2. Distribution of the probability density $q(e)$ for $d=2,34$.

The entangling power $e$ defines a random variable over $\mathcal{U}(\mathcal{H})$ if the latter, endowed with the Haar measure, is considered as a probability space. Therefore it makes sense to consider the associated density of probability distribution $q(e)$. Moreover, since the the manifold of unitary transformations over $\mathcal{H}$ is compact, the obviously continuous mapping $U \mapsto e(U)$ must achieves extrema, in particular $\exists \bar{U} \in \mathcal{U}(\mathcal{H}): e(\bar{U})=\max _{U} e(U)$. Such maximally entangling $\bar{U}$ 's will be referred to as optimal. In Fig. 2 are reported the $q(e)$ 's obtained numerically for the cases $d \times d$ with $d=2,3,4$. While in the cases $d \geq 2$ the function $q(e)$ vanishes on both the lower and the upper sides of the allowed range of $e$, it it remarkable that the two-qubit case $d=2$ shows a peculiar feature: $q(e)$ is a monotonic function of $e$. This implies that most of the two-qubit gates $U$ correspond to nearly optimal ones. Moreover, as will be discussed later in details, the entangling power of optimal $U$ 's does not correspond to an upper bound that is instead reached by all the other cases for $d$. In this sense the prototypical quantum information case of two qubits is quite singular. A first very natural question is how on the average an operator is entangling i.e., the mean of the $q(e)$.

Proposition 2 The average of the entangling power $e_{p_{0}}(U)$ over $U\left(d_{1} d_{2}\right)$ is given by

$$
{\overline{e_{p_{0}}(U)}}^{U}=\frac{\left(d_{1}-1\right)\left(d_{2}-1\right)}{d_{1} d_{2}+1}
$$

Proof. To prove Eq. (5) we first notice that, in view of definition (何), to compute the mean of the entangling power amounts to compute the average of the entanglement measure over all the states $|\Psi\rangle \in \mathcal{H}$ ( not just over the product states). The trace of the square of the reduced density matrix of $|\Psi\rangle$ is given by $\operatorname{tr}\left(|\Psi\rangle\left\langle\left.\Psi\right|^{\otimes 2} T_{13}\right)\right.$. Now we take the average with respect $|\Psi\rangle$ using again Lemma $\left[d_{1} d_{2}\left(d_{1} d_{2}+1\right)\right]^{-1} \operatorname{tr}\left[\left(\mathbb{1}+T_{13} T_{24}\right) T_{13}\right]=\left[d_{1} d_{2}^{2}+\right.$ $\left.d_{1}^{2}\right]\left[d_{1} d_{2}\left(d_{1} d_{2}+1\right)\right]^{-1}$. This expression inserted in the definition of the entanglement measure proves Eq. (5).

For proving bounds on the entangling power (11) it is useful to consider one of the states, say $\left|\psi_{2}\right\rangle$, of the input product as fixed. In this case a pair of CP-maps associated with $U$ are naturally defined. Indeed one has (explicit dependence on $U$ and $\left|\psi_{2}\right\rangle$ is omitted) $\Phi: \mathcal{S}\left(\mathcal{H}_{1}\right) \mapsto$ $\mathcal{S}\left(\mathcal{H}_{1}\right): \rho \mapsto \sum_{i=1}^{d_{2}} A_{i} \rho A_{i}^{\dagger}$ and $\tilde{\Phi}: \mathcal{S}\left(\mathcal{H}_{1}\right) \mapsto \mathcal{S}\left(\mathcal{H}_{2}\right): \rho \mapsto$ $\sum_{i=1}^{d_{1}} \tilde{A}_{i} \rho \tilde{A}_{i}^{\dagger}$ where $A_{i}: \mathcal{H}_{1} \mapsto \mathcal{H}_{1}$ and $\tilde{A}_{j}: \mathcal{H}_{1} \mapsto \mathcal{H}_{2}$ are given by $A_{i}:=\left\langle j|U| \psi_{2}\right\rangle, \quad\left(j=1, \ldots, d_{2}\right)$

$\tilde{A}_{i}:=\sum_{j=1}^{d_{2}}|j\rangle\langle i| A_{j}, \quad\left(i=1, \ldots, d_{1}\right)$. Therefore one can also define, for fixed $\left|\psi_{2}\right\rangle \in \mathcal{H}_{2}$ the (partial) entangling power of $U$ as $\tilde{e}_{p}(\Phi):=\overline{E(\Phi|\psi\rangle\langle\psi|)}^{\psi}$. Notice that the equation above can also be written is the form (3) with a special choice for $p\left(\psi_{1}, \psi_{2}\right)$ i.e., with $\Omega_{p}=\int \mu\left(\psi_{1}\right)\left(\left|\psi_{1}\right\rangle\left\langle\psi_{1}|\otimes| \psi_{2}\right\rangle\left\langle\psi_{2}\right|\right)^{\otimes 2}$. The definition of $\tilde{e}_{p}(\Phi)$ of course makes sense for general CPmaps, in this case the expression for $\tilde{e}_{p}(\Phi)$ analogous to Eq. (3) is given by $\tilde{e}_{p}(\Phi)=2 \operatorname{tr}\left[\Phi^{\otimes 2}\left(\omega_{p}\right) P_{13}^{-}\right]$where $\omega_{p}:=\int d \tilde{\mu}(\psi)|\psi\rangle\left\langle\left.\psi\right|^{\otimes 2} \in \mathcal{S}\left(\mathcal{H}_{1}^{\otimes 2}\right)\right.$.

Proposition 3 The entangling power of the CP-map $\Phi: \mathcal{S}\left(\mathcal{H}_{1}\right) \mapsto \mathcal{S}\left(\mathcal{H}_{1}\right)$ with respect to the uniform distribution is given by

$$
\tilde{e}_{p_{0}}(\Phi)=1-C_{d_{1}}\left(\operatorname{tr}_{2} \tilde{X}^{2}+\operatorname{tr}_{1} X^{2}\right)
$$

where $X:=\sum_{j=1}^{d_{2}} A_{i} A_{i}^{\dagger}$ and $\tilde{X}:=\sum_{j=1}^{d_{1}} \tilde{A}_{i} \tilde{A}_{i}^{\dagger}$.

Proof. One has that $\operatorname{tr}_{1} \rho^{2}$ is given by $\sum_{i, j=1}^{d_{2}} \operatorname{tr}_{1}\left(A_{i}\left|\psi_{1}\right\rangle\left\langle\psi_{1}\left|A_{i}^{\dagger} A_{j}\right| \psi_{1}\right\rangle\left\langle\psi_{1}\right| A_{j}^{\dagger}\right)$, this last expression can be rewritten as $\sum_{i, j=1}^{d_{2}} \operatorname{tr}_{1}\left[\left(A_{j}^{\dagger} A_{i}\right) \otimes\right.$ $\left(A_{i}^{\dagger} A_{j}\right)\left|\psi_{1}\right\rangle\left\langle\left.\psi_{1}\right|^{\otimes 2}\right]$. Now we perform the average with respect $\left|\psi_{1}\right\rangle$; using Lemma $\omega_{p_{0}}=C_{d_{1}}(\mathbb{1}+T)$. Using again the identity $\operatorname{tr}[(A \otimes B) T]=\operatorname{tr}(A B)$ one gets 


$$
C_{d_{1}}^{-1}{\overline{\operatorname{tr}_{1} \rho^{2}}}^{\psi_{1}}=\sum_{i, j=1}^{d_{2}}\left|\operatorname{tr}_{1}\left(A_{j}^{\dagger} A_{i}\right)\right|^{2}+\operatorname{tr}_{1}\left(\sum_{1=}^{d_{2}} A_{i} A_{i}^{\dagger}\right)^{2}
$$

[Notice that the two terms in the equation above corresponds to the $I_{\alpha}$ 's in Eq. (4).].] It is then straightforward algebra to check that the first term in the equation above can be written as $\operatorname{tr}_{2} \tilde{X}^{2}$

We now provide bounds on the entangling power. We assume that $d_{1} \leq d_{2}$.

Proposition 4 For any $U \in \mathcal{U}(\mathcal{H})$ one has

$$
0 \leq e_{p_{0}}(U) \leq \frac{d_{2}-d_{2} / d_{1}}{d_{2}+1} .
$$

Proof. The lower bound is obvious in view of the definition (1), it is achieved by all the unitaries obtained composing bi-local transformations of $U(d) \times U(d)$ with the swap. Let us consider first the operator $X$ in Eq. (6). By denoting with $\Phi$ the CP-map associated with the $A_{j}$ 's one has $\Phi\left(\mathbb{1} / d_{1}\right)=X / d_{1}$ and then $1-\operatorname{tr}_{1} \Phi\left(\mathbb{1} / d_{1}\right)^{2} \leq 1-1 / d_{1}$ (general bound on linear entropy) it follows that $d_{1} \leq \operatorname{tr}_{1} X^{2}$. This latter inequality provides a bound on the second term of Eq. (6). Reasoning in the same way with the operator $\tilde{X}$ and the associated CP-map $\tilde{\Phi}$ one finds for the first term of (6) the lower bound $d_{1}^{2} / d_{2}$. Putting these two results together, and in view of the assumption $d_{1} \leq d_{2}$, inverting $d_{1}$ with $d_{2}$ one gets the desired result (8)

Another issue is to understand whether the upper bound in Eq. (8) is achieved by an optimal unitary transformation $U$. As we shall show in the following the answer seems to be affirmative for $d>2$, Let us stress that this is not obvious at all in that the upper bound (8) has been obtained by providing separate bounds on the two terms appearing in $e_{p_{0}}(U)$. Notice that the proof of Proposition 3 allows us to state the condition on $U$ in order to saturate the bound (8) as : for any initial state $|\psi\rangle \in \mathcal{H}_{1}$ the associated CP-maps $\Phi$ and $\tilde{\Phi}$ (depending both on $|\psi\rangle$ and $U)$ must be unital i.e., they map totally mixed states onto totally mixed states. It might well be that no $U$ 's yields unitality for both CP-maps at once. This in fact turns out numerically to be the case for $d=2$, in which one has that the optimal $U$ 's are such that [see Fig. 2] $e_{p_{0}}(U)=2 / 9<1 / 3$. An optimal operators for qubit is given (not surprisingly) by the controlled-not $U:=|0\rangle\langle 0|\otimes \mathbb{1}+| 1\rangle\langle 1| \otimes X$, where $X:=|0\rangle\langle 1|+| 1\rangle\langle 0|$. More interestingly the operators providing a natural $d$ dimensional generalization of the controlled-not are in general not optimal. This is shown by the following calculation.

Let us consider, for $d_{1}=d_{2}, U=\sum_{\alpha=1}^{d}|\alpha\rangle\langle\alpha| \otimes U_{\alpha}$, where the $|\alpha\rangle$ 's are a $d$-dimensional orthonormal basis and the $U_{\alpha}$ 's are unitaries which, without any loss of generality can be taken to be orthogonal with respect to the Hilbert-Schmidt scalar product i.e., $\left\langle U_{\alpha}, U_{\beta}\right\rangle=d \delta_{\alpha, \beta}$. By using Eq. (4) is easy to prove that for these unitaries one has $e_{p_{0}}(U)=d(d-1) /(d+1)^{2}$, that is $d /(d+1)$ times smaller than the bound (8) and, for $d>2$ even smaller.

We also performed numerical maximization of $e_{p_{0}}(U)$ trying to find exact expressions for the optimal unitary transformations. For $d_{1}=d_{2}=d=$ odd we have found that the following "classical" unitary transformation (which only permutes the $d^{2}$ bases states) is optimal and reaches the bound: $U|i\rangle \otimes|j\rangle=|i+j\rangle \otimes|i-j\rangle$ where $i, j=0 \ldots d-1$ and the sums are $\bmod d$. [Notice that the above expression for even $d$ does not define a permutation of the basis of $\mathcal{H}$.] Similarly a more complicated construction gives an optimal permutation that achieves the bound for the case $d_{1}=d_{2}=d=4 n$. Thus for equal dimensions the only case that remains to be solved is $d_{1}=d_{2}=d=4 n+2$, e.g. $6 \times 6$. We have also found unitary transformations satisfying the bound for a very asymmetric case, namely $d_{2}=n \cdot m$ with $n, m \geq d_{1}$. This last example is of the type of controlled unitary operation from the larger to the smaller system. The previous constructions for equal dimensions can be viewed as a concatenation of two such controlled operations, in which the control role is played alternatively by one of the subsystems. It may also be interesting that for the cases $2 \times$ odd the bound can be shown not to be reached by permutations.

Let us finally discuss briefly the numerical evidences. First, even for dimensions $2 \times 3$ the bound appears not to be reached, rather for optimal $U$ 's we get the value $1 / 3$ (instead of 3/8). For all other cases that we have checked the bound seems to be reached, namely for $2 \times 4$ up to $2 \times 7$, and $3 \times 4$ up to $3 \times 6$. In conclusion one might conjecture that the only cases where the optimal transformations do not reach the bound (8) are $2 \times 2$ and $2 \times 3$ [8].

Conclusions. In this paper we introduced a measure for the entangling power $e(U)$ of unitary transformations $U$ acting on the state-space $\mathcal{H}$ of a bi-partite $d_{1} \times d_{2}$ quantum system. In terms of this measure we moved a first step towards the analysis of the manifold of bi-partite unitary transformations. We analyzed the induced probability distribution $q(e)$ over $\mathcal{U}(\mathcal{H})$ as $d_{1}$ and $d_{2}$ varies, and we found an analytical form of optimal transformations for some cases. Although we believe that both the questions addressed and the approach we adopted are quite natural and physically motivated the role, if any, that the entangling power will play in Quantum Information theory is still an issue for future work.

The authors thank M. Rasetti and J. Pachos for useful discussions. Ch. Z. is supported by the EU project IST-Q-ACTA. 
[1] For reviews, see D.P. DiVincenzo and C. Bennet Nature 404, 247 (2000); A. Steane, Rep. Prog. Phys. 61, 117 (1998)

[2] D. Deutsch, A. Barenco and A. Ekert, Proc. R. Soc. London A, 449, 669 (1995); D.P. Di Vincenzo, Phys. Rev. A, 50, 1015 (1995)

[3] S. Bose et al Communication Capacity of Quantum Computation, quant-ph/0003072

[4] K. Kraus, "States, Effects, and Operations: Fundamental Notions of Quantum Theory", Lecture Notes in Physics, 190, Springer, Berlin (1983)

[5] L.M. Duan and G.C. Guo, Phys. Rev. Lett, 79, 1953
(1997); P. Zanardi and M. Rasetti, Phys. Rev. Lett. 79, 3306 (1997);

[6] The subsystems composing our bi-partite system could be on their own multipartite. In this case most of the $\left|\psi_{i}\right\rangle$ 's $(i=1,2)$ will be entangled, and in this sense "difficult" to produce.

[7] J.F. Cornwell, Group Theory in Physics (Academic, New York, 1984), Vol. I

[8] Since our constructions for achieving the bound have a rather number theoretic character it is still imaginable that there are more exceptions at specific higher dimensions. 